\title{
Strong lower energy estimates for nonlinearly damped Timoshenko beams and Petrowsky equations
}

\author{
Fatiha Alabau-Boussouira
}

\begin{abstract}
The purpose of this paper is to establish strong lower energy estimates for strong solutions of nonlinearly damped Timoshenko beams, Petrowsky equations in two and three dimensions and wave-like equations for bounded one-dimensional domains or annulus domains in two or three dimensions. We also establish weak lower velocity estimates for strong solutions of the nonlinearly damped Petrowsky equation in two and three dimensions. The feedbacks in consideration have arbitrary growth close to the origin. These results improve the strong lower energy decay rates obtained in our previous papers (Alabau-Boussouira in J Differ Equ 249:1145-1178, 2010; J Differ Equ 248:1473-1517, 2010) for strong solutions of the nonlinearly locally damped wave equation and extend to systems and to Petrowsky equation the method of Alabau-Boussouira (J Differ Equ 249:1145-1178, 2010; J Differ Equ 248:1473-1517, 2010). These results are the first ones for Timoshenko beams and Petrowsky equations.
\end{abstract}

Mathematics Subject Classification (2000). 34G10, 35B35, 35B37, 35L90, 93D15, 93D20.

Keywords. Timoshenko beams, Wave equation, Petrowsky equation, Nonlinear dissipation, Boundary damping, Locally distributed feedback, Hyperbolic equations, Optimality, Asymptotic behavior, Lower energy estimates, Regularity, Energy comparison principles.

\section{Introduction}

Damped reversible systems are involved in many applications in engineering, mechanics and acoustics. They have been the subject of wide investigations in

F. Alabau-Boussouira's research was partially supported by the GDRE-CNRS-INDAM CONEDP. 
mathematics. If strong stabilization and upper energy estimates have been a lot explored, comparatively very few is known on lower energy estimates and optimality in case of nonlinear stabilization.

Vancostenoble [37] and Vancostenoble and Martinez [36] proved optimality of the upper energy estimates for a one dimensional (or radial in three dimensions) boundary damped wave equation and for initial data with vanishing velocities.

Haraux [14] considered a one-dimensional locally polynomially damped wave equation. He proved a weak (in the sense of a certain lim sup) lower velocity estimate from which he derived a weak energy estimate for initial data in $W^{2, \infty}(\Omega) \times W^{1, \infty}(\Omega)$.

We showed in [1] that it is possible to derive strong lower energy estimates for general dampings, localized as well as boundary dampings under weaker regularity assumptions on the initial data. We also extended the weak lower velocity estimates of Haraux to general dampings and multidimensional domains for bounded velocities in time and space.

The purpose of this paper is to give an extension of our method, to a system, namely the Timoshenko beams and to Petrowsky equation in two and three dimensions. We also prove weak lower velocity estimates for strong solutions of the Petrowsky equation in two and three dimensions. Further, we improve the strong lower bound of [1] for nonlinearly locally damped wave equation in one dimensional domains or in annulus domains for two and three space dimensions. Our method relies on energy comparison principles introduced for the first time in $[1,2]$ (for finite as well as infinite dimensional systems) and elementary interpolation properties. It also uses Dafermos' strong stabilization result and Lasalle invariance principle combined with unique continuation properties.

The Lyapunov type method used in [14] to prove $L^{\infty}$ regularity and a priori estimates of the velocities of locally polynomially damped one-dimensional wave equations is specific to the structure of locally damped wave equations in one-dimension. It does not extend to boundary damped wave equations, nor to Petrowsky equation nor to systems such as Timoshenko beams.

Our method extends to systems and as well to higher order equation in space, such as the Petrowsky equation. In this latter case, the problem of smoothness and a priori estimate on the velocity in spaces is solved thanks to Sobolev embeddings and appropriate Gagliardo-Nirenberg inequalities, which allows us to give the first lower energy estimates for Petrowsky equation in two or three dimensions. We also derive weak lower velocity estimates in this latter case.

We already show in [1] that our method also works for boundary general nonlinear dampings for wave equations in one dimensional, or annulus domains in two and three dimensions.

Thus, an important issue is that our method is general regarding several aspects such as feedback growth, localized or boundary feedbacks, and is applicable to different PDE's. It also raises new challenges or shows the importance of still unsolved ones - such as regularity of the velocity-, which 
are interesting both in themselves and in the framework of lower estimates and optimality. These challenges concern the regularity of solutions - in particular of the velocity - , the behavior of higher order energies, the determination of the asymptotic behavior of the solutions depending on initial data and the possible dependence of the asymptotic behavior on the spatial dimension and the geometry of the domain.

We recall that Dafermos [11] establishes strong stabilization, i.e. the energy of solutions converges to zero as time goes to infinity, for a nonlinearly locally damped wave equation provided that the damping is effective on a non empty open set (see [16] for a generalization to monotone graphs dampings). His proof is based on Lasalle invariance principle [20,21] and on the dissipativity properties of the energy and on the energy of first order, i.e. the energy of the time derivative of the solution. Dafermos pointed in this paper the lack of information on the rate of decay.

Since then, many upper energy estimates have been derived at first "polynomially" growing feedbacks (see $[9,14,17,19,41,33]$ and the references therein), and then for arbitrary growing feedbacks [1,2,5,6,12,22,27-29] and the references therein). We also refer to Zuazua [40] and to [13] for exponential stabilization of linearly locally damped semilinear wave equations and to Coron [10] for results on nonlinear systems (and the references therein).

Let us further mention that the question of lower energy estimates for arbitrary domains in dimensions two or three is still open, as well as the optimality of upper energy estimates derived in [2,5,12,22,27-29]. As above written, a first step in this direction has been obtained by Vancostenoble [36] and Vancostenoble and Martinez [36] in the case of a one dimensional (or radial in three dimensions) boundary damped wave equation and for initial data with vanishing velocities. Carpio [8] obtained results concerning the dependence of the estimates with respect to initial data for power-like nonlinearities.

Remark 1.1. In all the paper, we will consider initial data with nonvanishing energy. The lower estimates resulting in the case of null energy initial data are trivial (the lower bound is 0 in this case). This means that in this trivial case, the nonnegative constant, which depends on the initial data, involved in factor of the lower estimate is vanishing, whereas it is positive if the initial data have nonzero energy.

The paper is organized as follows. After a brief introduction, we present the strong lower energy estimates for Timoshenko beams in Sect. 2. Section 3 is devoted to strong lower energy and weak lower velocity estimates for Petrowsky equation in two and three dimensions. Sections 4 and respectively 5 are devoted to the improvements of our lower energy estimates of [1] for the one dimensional wave equation and respectively the wave equation in annulus domains in dimensions 2 and 3. We conclude and present a series of open problems connected to lower energy estimates, regularity of the velocity and optimality in Sect. 6. 


\section{Lower energy estimates for Timoshenko beams}

We consider the following Timoshenko type system (see e.g. [7,31,34]):

$$
\begin{cases}\rho_{1} \varphi_{t t}-k\left(\varphi_{x}+\psi\right)_{x}=0 & t>0,0<x<L, \\ \rho_{2} \psi_{t t}-b \psi_{x x}+k\left(\varphi_{x}+\psi\right)+a(x) g\left(\psi_{t}\right)=0 t>0, & 0<x<L\end{cases}
$$

where $a \in L^{\infty}(\Omega)$ and $a \geq 0$ a.e. on $\Omega$ with $a>0$ on an open subset $\omega$ of $\Omega$. We set $\Omega=(0, L)$. We also make the following assumptions on $g$ in the sequel

$$
(H 1)\left\{\begin{array}{l}
g: \mathbb{R} \mapsto \mathbb{R} \text { is assumed to be an odd, increasing } \\
\text { continuously differentiable function } \\
g \text { has a linear growth at infinity, } \\
s g(s)>0 \quad \forall s \in \mathbb{R}^{\star} \\
g(0)=g^{\prime}(0)=0 .
\end{array}\right.
$$

Remark 2.1. We assume that $g$ is a $\mathcal{C}^{1}$ function on $\mathbb{R}$. This assumption can be relaxed with no difficulty. It suffices that $g$ has this regularity in a neighbourhood of 0 . We prefer to shorten the formulation of the results, so that the exposition is simple and clear.

Indeed, as soon as one assumes that $g$ has a linear growth at infinity, then the upper asymptotic behavior of the energy as times goes to $\infty$ is determined by the behavior of $g$ close to 0 as shown in $[2,5,22,27,29]$. Hence, the values of $g$ outside a neighbourhood of $g$ does not affect the behavior of the energy at $\infty$.

The functions $\varphi$ and $\psi$ denote respectively the transverse displacement of the beam and the rotation angle of the filament. The term $a g\left(\psi_{t}\right)$ is the damping term, so one can remark that only the second equation is damped whereas the first equation is not damped. Here $g$ is assumed to satisfy $(H 1)$. Moreover, $\rho_{1}, \rho_{2}, k$ and $b$ denote positive constants characterizing physical properties of the beam and the filament. The speeds of propagation in the first and second equations are respectively given by

$$
v_{1}=\frac{k}{\rho_{1}}
$$

and

$$
v_{2}=\frac{b}{\rho_{2}}
$$

We consider two types of boundary conditions for this system, namely

$$
\begin{aligned}
& \varphi=\psi_{x}=0, t>0, x=0, x=L, \\
& \varphi=\psi=0, t>0, x=0, x=L .
\end{aligned}
$$


Moreover the initial conditions for the state variables are:

$$
\left\{\begin{array}{l}
(\varphi, \psi)(x, 0)=\left(\varphi^{0}(x), \psi^{0}(x)\right) \quad x \in(0, L) \\
\left(\varphi_{t}, \psi_{t}\right)(x, 0)=\left(\varphi^{1}(x), \psi^{1}(x)\right) \quad x \in(0, L) .
\end{array}\right.
$$

The energy of solutions of the system (2.1) subjected to initial state (2.6) and to either the boundary conditions (2.4) or (2.5) is defined by

$$
E(t)=\frac{1}{2} \int_{0}^{L}\left(\rho_{1} \varphi_{t}^{2}+\rho_{2} \psi_{t}^{2}+b \psi_{x}^{2}+k\left|\varphi_{x}+\psi\right|^{2}\right) d x .
$$

Well-posedness of these two systems together with regularity of solutions and of the energy for smoother initial data is considered in $[3,7,31]$.

For the moment, we can remark that if we multiply formally the first equation in (2.1) by $\varphi_{t}$, the second one by $\psi_{t}$ and if we add the two resulting equations, we obtain the following dissipation relation

$$
E^{\prime}(t)=-\int_{0}^{L} a(x) \psi_{t} g\left(\psi_{t}\right) d x \leq 0 .
$$

We set $U^{0}=\left(\varphi^{0}, \psi^{0}, \varphi^{1}, \psi^{1}\right)$ and denote by $U=U(t)=\left(\varphi, \psi, \varphi_{t}, \psi_{t}\right)$ the solution of either (2.1), (2.4), (2.6) or (2.1), (2.5), (2.6).

We denote by $E_{1}(t)$ the energy defined by (2.7), where $\varphi$ and $\psi$ are replaced by $\varphi_{t}$ and $\psi_{t}$ and where $U^{\prime}(t)=\left(\varphi_{t}, \psi_{t}, \varphi_{t t}, \psi_{t t}\right) . E_{1}$ is the energy of first order.

Remark 2.2. We assume above that $g$ is a globally Lipschitz function on $\mathbb{R}$. This assumption can be removed as far as lower energy estimates are concerned at least to include power like nonlinearities $g$ of the form $g(s)=|s|^{p-1} s$ for $s \in \mathbb{R}$, under conditions on $p$.

We define the energy space associated to problem (2.1), (2.4), (2.6) by

$$
\mathcal{H}_{1}=H_{0}^{1}(\Omega) \times H^{1}(\Omega) \times\left(L^{2}(\Omega)\right)^{2},
$$

and the unbounded operator in $\mathcal{H}_{1}$ defined by

$$
\begin{aligned}
& D\left(A_{1}\right)=\left(H_{0}^{1}(\Omega) \cap H^{2}(\Omega)\right) \times H^{2}(\Omega) \times H_{0}^{1}(\Omega) \times H^{1}(\Omega), \\
& \quad \text { for } U=(\varphi, \psi, w, z) \in D\left(A_{1}\right), \\
& A_{1} U=\left(w, z, \frac{k}{\rho_{1}}\left(\varphi_{x x}+\psi_{x}\right), \frac{b}{\rho_{2}} \psi_{x x}-\frac{k}{\rho_{2}}\left(\varphi_{x}+\psi\right)\right) .
\end{aligned}
$$

We also define the damping nonlinear operator

$$
B U=\left(0,0,0,-\frac{a}{\rho_{2}} g(z)\right) .
$$

In a similar way, we define the energy space associated to problem (2.1), (2.5), (2.6) by

$$
\mathcal{H}_{2}=\left(H_{0}^{1}(\Omega)\right)^{2} \times\left(L^{2}(\Omega)\right)^{2},
$$


and the unbounded operator in $\mathcal{H}_{2}$ defined by

$$
\begin{aligned}
& D\left(A_{2}\right)=\left(H_{0}^{1}(\Omega) \cap H^{2}(\Omega)\right)^{2} \times\left(H_{0}^{1}(\Omega)\right)^{2} \\
& \quad \text { for } U=(\varphi, \psi, w, z) \in D\left(A_{2}\right), \\
& \quad A_{2} U=\left(w, z, \frac{k}{\rho_{1}}\left(\varphi_{x x}+\psi_{x}\right), \frac{b}{\rho_{2}} \psi_{x x}-\frac{k}{\rho_{2}}\left(\varphi_{x}+\psi\right)\right) .
\end{aligned}
$$

The nonlinear damping operator $B$ is defined as above.

Problem $(2.1),(2.4),(2.6)$ can be reformulated in the abstract equation

$$
\begin{aligned}
& U^{\prime}(t)=A_{1} U(t)+B U(t) \quad t>0 . \\
& U(0)=U^{0}=\left(\varphi^{0}, \psi^{0}, \varphi^{1}, \psi^{1}\right) \in \mathcal{H}_{1} .
\end{aligned}
$$

In a similar way, problem $(2.1),(2.5),(2.6)$ can be reformulated in the abstract equation

$$
\begin{aligned}
& U^{\prime}(t)=A_{2} U(t)+B U(t) \quad t>0 . \\
& U(0)=U^{0}=\left(\varphi^{0}, \psi^{0}, \varphi^{1}, \psi^{1}\right) \in \mathcal{H}_{2} .
\end{aligned}
$$

We recall the following classical existence and regularity result (see e.g. [15] for the proof) using the theory of maximal nonlinear monotone operator:

Theorem 2.3. Assume that $g$ satisfies $(H 1)$. Let $i=1$ or 2 . Then for all $\left(\varphi^{0}, \psi^{0}, \varphi^{1}, \psi^{1}\right)$ in $\mathcal{H}_{i}$, the problem (2.1) and (2.6) subjected to (2.4) if $i=1$, and $(2.5)$ if $i=2$, has a unique solution $U \in \mathcal{C}\left([0,+\infty) ; \mathcal{H}_{i}\right)$. For $i=1,2$, the operator $A_{i}+B$ generates a continuous semigroup $\left(\mathcal{T}_{i}(t)\right)_{t \geq 0}$ on $\mathcal{H}_{i}$. Moreover, for all $\left(\varphi^{0}, \psi^{0}, \varphi^{1}, \psi^{1}\right)$ in $D\left(A_{i}\right)$, the solution $U$ is in $L^{\infty}\left([0,+\infty) ; D\left(A_{i}\right)\right) \cap$ $W^{1, \infty}\left([0,+\infty) ; \mathcal{H}_{i}\right)$ and its energy defined by $(2.7)$ satisfies the dissipation relation (2.8). Moreover $E_{1}$, the energy of first order, is nonincreasing.

To recall Dafermos'principle applied to Timoshenko system, we need to consider the conservative system corresponding to (2.1), that is

$$
\begin{cases}\rho_{1} \varphi_{t t}-k\left(\varphi_{x}+\psi\right)_{x}=0 & t>0, \quad 0<x<L \\ \rho_{2} \psi_{t t}-b \psi_{x x}+k\left(\varphi_{x}+\psi\right)=0 & t>0, \quad 0<x<L\end{cases}
$$

subjected to either (2.4) or (2.5).

We say in all the sequel, that a subset $\omega$ of $\Omega$ satisfies the assumption $(H S)$ below if it is such that

$(H S)\left\{(\varphi, \psi)\right.$ is a weak solution of $(2.9)$ and $\psi_{t} \equiv 0$ on $\omega \Longrightarrow(\varphi, \psi) \equiv(0,0)$

Theorem 2.4. Assume the hypotheses of Theorem 2.3. We assume in addition that $\omega$ satisfies $(H S)$. Then for all $U_{0}=\left(\varphi^{0}, \psi^{0}, \varphi^{1}, \psi^{1}\right)$ in $\mathcal{H}_{i}$, the solution of (2.1) and (2.6) subjected to (2.4) if $i=1$ and to (2.5) if $i=2$, is such that its energy $E$ defined by (2.7) satisfies

$$
\lim _{t \rightarrow \infty} E(t)=0 .
$$

Proof. The proof is standard. We adapt and apply Dafermos' [11] strong stabilization technique based on Lasalle invariance principle. We detail the steps for the reader convenience only in the case of boundary conditions (2.5). The proof can easily be adapted to the case of boundary conditions (2.4). We 
first assume that the initial data $U_{0}=\left(\varphi^{0}, \psi^{0}, \varphi^{1}, \psi^{1}\right)$ are in $D\left(A_{2}\right)$. We set $U=(\varphi, p, \psi, q)=\mathcal{T}_{2}(t) U_{0}$ and define $V$ on $\mathcal{H}_{2}$ by

$$
V(U)=\frac{1}{2} \int_{0}^{L}\left(\rho_{1} p^{2}+\rho_{2} q^{2}+b \psi_{x}^{2}+k\left|\varphi_{x}+\psi\right|^{2}\right) d x .
$$

Then thanks to the dissipation relation on $E, V$ is a Liapunov function for $\mathcal{T}_{2}(t)$. Moreover, thanks to Theorem 2.3, the energy of order 1 is nonincreasing so that

$$
E_{1}(t)=\frac{1}{2} \int_{0}^{L}\left(\rho_{1} \varphi_{t t}^{2}+\rho_{2} \psi_{t t}^{2}+b \psi_{t x}^{2}+k\left|\varphi_{t x}+\psi_{t}\right|^{2}\right) d x \leq E_{1}(0), \quad \forall t \geq 0 .
$$

Hence, we have

$$
E_{1}(t) \leq E_{1}(0) \quad \forall t \geq 0 .
$$

Thanks to the definition of $E_{1}$, we deduce that the sets $\left\{\psi_{t t}(t,),. t \geq 0\right\}$ and $\left\{\psi_{t}(t,),. t \geq 0\right\}$ are bounded respectively in $L^{2}(\Omega)$ and in $H_{0}^{1}(\Omega)$. Hence the set $\left\{\psi_{t}(t,),. t \geq 0\right\}$ is relatively compact in $L^{2}(\Omega)$. Similarly, using in addition the fact that $E$ is bounded uniformly on $\mathbb{R}^{+}$, we deduce easily that the sets $\left\{\varphi_{t t}(t,),. t \geq 0\right\}$ and $\left\{\varphi_{t}(t,),. t \geq 0\right\}$ are bounded respectively in $L^{2}(\Omega)$ and in $H_{0}^{1}(\Omega)$. Hence the set $\left\{\varphi_{t}(t,),. t \geq 0\right\}$ is relatively compact in $L^{2}(\Omega)$. Using now (2.1), we deduce that $\left\{\psi_{x x}(t,),. t \geq 0\right\}$ and $\left\{\varphi_{x x}(t,),. t \geq 0\right\}$ are bounded in $L^{2}(\Omega)$. Thus, the sets $\{\psi(t,),. t \geq 0\}$ and $\{\varphi(t,),. t \geq 0\}$ are relatively compact in $H_{0}^{1}(\Omega)$, whereas the sets $\left\{\psi_{t}(t,),. t \geq 0\right\}$ and $\left\{\varphi_{t}(t,),. t \geq 0\right\}$ are relatively compact in $L^{2}(\Omega)$. We denote by $\gamma\left(U_{0}\right)=\cup_{t>0} \mathcal{T}_{2}(t) U_{0}$ the orbit of $U_{0}$. Thus $\gamma\left(U_{0}\right)$ is relatively compact in $\mathcal{H}_{2}$. We denote the $\omega$-limit set of $U_{0}$ by $\omega\left(U_{0}\right)$. Using then Lasalle invariance principle as in [11] (see also [20,21]), we deduce that for each $W_{0} \in \omega\left(U_{0}\right)$, the map $t \mapsto V\left(\mathcal{T}_{2}(t) W_{0}\right)$ is constant. Let now $Z_{0} \in \omega\left(U_{0}\right)$ be given and set $Z(t)=(w, r, z, \theta)(t)=\mathcal{T}_{2}(t) Z_{0}$. Since $V(Z()$.$) is constant, we deduce that (w, z)$ is solution of the system

$$
\left\{\begin{array}{lcc}
\rho_{1} w_{t t}-k\left(w_{x}+z\right)_{x}=0 & t>0, & 0<x<L, \\
\rho_{2} z_{t t}-b z_{x x}+k\left(w_{x}+z\right)=0 & t>0, & 0<x<L, \\
\left(w(0), w_{t}(0), z(0), z_{t}(0)\right)=Z_{0} & \text { in } \Omega, \\
z_{t}=0 \text { on }\{x \in \Omega, a(x) \neq 0\} \supset \omega, &
\end{array}\right.
$$

subjected to $(2.5)$. Thanks to $(H S)$, we deduce that $(w, z) \equiv(0,0)$. Hence $\omega\left(U_{0}\right)=\{(0,0,0,0)\}$, so that since the orbit is relatively compact in $\mathcal{H}_{2}$, the energy $E(t)$ associated to the initial data $U_{0}$ converges to 0 as time goes to $\infty$. Thanks to the dissipation relation for $E$ and since $D\left(A_{2}\right)$ is dense in $\mathcal{H}_{2}$, we easily conclude that $E(t)$ converges to 0 as $t$ goes to $\infty$ for initial data in $\mathcal{H}_{2}$.

We now come to the lower energy estimates using the method developed for strong solutions in [1] (see also [2] for first partial results in this direction). For this, we define functions $H, \widetilde{H}$ and $\Lambda$ as follows

$$
H(x)=\sqrt{x} g(\sqrt{x}), \quad x \geq 0
$$


and

$$
\begin{aligned}
\widetilde{H}(x) & =\frac{H(x)}{x}, \quad x>0, \quad \widetilde{H}(0)=0, \\
\Lambda(x) & =\frac{H(x)}{x H^{\prime}(x)} .
\end{aligned}
$$

We can establish a lower bound of the energy using comparison principles expressed through the energy of the solutions as in [2].

Theorem 2.5. Assume the hypotheses of Theorem 2.3. Let $U_{0}=\left(\varphi^{0}, \psi^{0}, \varphi^{1}, \psi^{1}\right)$ be in $D\left(A_{i}\right)$. We denote by $U$ the solution of (2.1) and (2.6) subjected to (2.4) if $i=1$ and to (2.5) if $i=2$, and $E$ its energy. We assume in addition that either $\omega$ satisfies $(H S)$ or that $\lim _{t \longrightarrow \infty} E(t)=0$. We define the functions $H$, and $\widetilde{H}$ respectively by (2.11) and (2.12). We assume that $\widetilde{H}$ is nondecreasing on $\left[0, r_{0}^{2}\right]$ for $r_{0}>0$ sufficiently small. Then there exists $T_{0} \geq 0$, depending on $E_{1}(0)$ such that defining $K_{s}$ by

$$
K_{s}(\tau)=\int_{\tau}^{\gamma_{s} \sqrt{E\left(T_{0}\right)}} \frac{1}{H(y)} d y, \quad \tau \in\left(0, \gamma_{s} \sqrt{E\left(T_{0}\right)}\right]
$$

E satisfies the lower estimate

$$
\left(\frac{1}{\gamma_{s}} K_{s}^{-1}\left(\frac{c \alpha_{a}}{\rho_{2}}\left(t-T_{0}\right)\right)\right)^{2} \leq E(t), \quad \forall t \geq T_{0},
$$

where $\alpha_{a}$ is defined in (2.23) and $\gamma_{s}$ is defined later in (2.17) if (2.4) holds or by (2.19) if (2.5) holds.

Moreover if $\lim _{\tau \rightarrow 0^{+}} K_{s}(\tau)=\infty$, then

$$
\lim _{t \rightarrow \infty} K_{s}^{-1}\left(\frac{c \alpha_{a}}{\rho_{2}}\left(t-T_{0}\right)\right)=0,
$$

so that the left hand side of (2.15) is converging to 0 as time goes to $\infty$.

Proof. We first consider the boundary conditions (2.4). Then, thanks to the smoothness of the solutions, we have

$$
\begin{aligned}
\psi_{t}^{2}(t, x)= & \psi_{t}^{2}(t, y)+2 \int_{y}^{x} \psi_{t}(t, z) \psi_{t x}(t, z) d z \leq \psi_{t}^{2}(t, y) \\
& +2 \sqrt{\int_{0}^{L} \psi_{t}^{2} d x} \sqrt{\int_{0}^{L} \psi_{t x}^{2} d x} \quad \forall t \geq 0, \quad x \in(0, L) .
\end{aligned}
$$

Integrating this inequality with respect to $y$, we obtain

$$
\begin{aligned}
\psi_{t}^{2}(t, x) & \leq \frac{1}{L} \int_{0}^{L} \psi_{t}^{2}(x)+2 \sqrt{\int_{0}^{L} \psi_{t}^{2} d x} \sqrt{\int_{0}^{L} \psi_{t x}^{2} d x} \\
& \leq \gamma_{s} \sqrt{E(t)} \quad \forall t \geq 0, x \in(0, L)
\end{aligned}
$$

where

$$
\gamma_{s}=\frac{2}{\rho_{2}}\left(\frac{1}{L} \sqrt{E(0)}+2 \sqrt{E_{1}(0)}\right) \quad \text { if }(2.4) \text { holds }
$$


If we now consider the boundary condition (2.5), we have

$$
\psi_{t}^{2}(t, x) \leq \gamma_{s} \sqrt{E(t)} \quad \forall t \geq 0, \forall x \in(0, L),
$$

where

$$
\gamma_{s}=\frac{4}{\rho_{2}} \sqrt{E_{1}(0)} \quad \text { if }(2.5) \text { holds. }
$$

For both sets of boundary conditions, we have therefore

$$
\left\|\psi_{t}^{2}(t, .)\right\|_{L^{\infty}(\Omega)} \leq \gamma_{s} \sqrt{E(t)} \quad \forall t \geq 0,
$$

where $\gamma_{s}$ is defined as above.

If $E(t)$ converges to 0 when $t$ tends to $\infty$ or if $(H S)$ holds, then thanks to Theorem 2.4, there exists $T_{0} \geq 0$ such that

$$
E(t) \leq\left(\frac{r_{0}^{2}}{\gamma_{s}}\right)^{2}, \quad \forall t \geq T_{0}
$$

Thanks to (2.8), we have

$$
-E^{\prime}(t)=\int_{0}^{L} a(x) \psi_{t} g\left(\psi_{t}\right) d x \leq c \alpha_{a} \int_{0}^{L} \psi_{t}^{2} \widetilde{H}\left(\psi_{t}^{2}\right) d x \quad \forall t \geq T_{0},
$$

where $\alpha_{a}$ is defined by

$$
\alpha_{a}=\|a\|_{L^{\infty}(\Omega)} .
$$

Thanks to our hypotheses, $\widetilde{H}$ is increasing on $\left[0, r_{0}^{2}\right]$, therefore we have

$$
\widetilde{H}\left(\left|\psi_{t}^{2}(t, .)\right|\right) \leq \widetilde{H}\left(\gamma_{s} \sqrt{E(t)}\right) \quad \forall t \geq T_{0}, x \in(0, L) .
$$

Using this result in (2.22), we derive

$$
-E^{\prime}(t) \leq \frac{2 c \alpha_{a}}{\rho_{2} \gamma_{s}} \sqrt{E(t)} H\left(\gamma_{s} \sqrt{E(t)}\right), \quad \forall t \geq T_{0}
$$

Thus we deduce that

$$
K_{s}\left(\gamma_{s} \sqrt{E(t)}\right) \leq \frac{c \alpha_{a}\left(t-T_{0}\right)}{\rho_{2}}, \quad \forall t \geq T_{0}
$$

Since $K_{s}$ is nonincreasing, we obtain (2.15).

Remark 2.6. It is important to note that Haraux' proof of regularity and a priori estimates in $L^{\infty}\left([0, \infty), W^{1, \infty}(\Omega) \cap H^{1}(\Omega)\right)$ of $\psi_{t}$ does not extend to systems such as Timoshenko systems. It is requested for a sharper lower estimate as seen in [1]. Hence a method such as in [1] requesting less regularity assumptions and based only on the dissipation of the energy and of first order energy is useful for applications and extension to coupled systems. It also apply to a wider class of initial data, the counterpart being that the lower estimates are less sharp than the one derived in [1] for more regular solutions.

This above lower estimate can be characterized equivalently through pointwise time estimates, under some additional assumptions on $g$. For this, we need to use some of our results in [2], in particular Lemma 2.4, that we include here for the sake of completeness. 
Lemma 2.7. Let $G$ be a given strictly convex $\mathcal{C}^{1}$ function from $\left[0, r_{0}^{2}\right]$ to $\mathbb{R}$ such that $G(0)=G^{\prime}(0)=0$, where $r_{0}>0$ is sufficiently small and define $\widehat{\Lambda}$ on $\left(0, r_{0}^{2}\right]$ by

$$
\widehat{\Lambda}(x)=\frac{G(x)}{x G^{\prime}(x)} .
$$

Let $z$ be the solution of the ordinary differential equation:

$$
z^{\prime}(t)+\kappa G(z(t))=0, \quad z(0)=z_{0} \quad t \geq 0,
$$

where $z_{0}>0$ and $\kappa>0$ are given. Then $z(t)$ is defined for every $t \geq 0$ and decays to 0 at infinity. Moreover assume that either

$$
0<\liminf _{x \rightarrow 0} \widehat{\Lambda}(x) \leq \limsup _{x \rightarrow 0} \widehat{\Lambda}(x)<1
$$

or that there exists $\mu>0$ such that

$$
0<\liminf _{x \rightarrow 0}\left(\frac{G(\mu x)}{\mu x} \int_{x}^{z_{1}} \frac{1}{G(y)} d y\right) \text {, and } \limsup _{x \rightarrow 0} \widehat{\Lambda}(x)<1,
$$

for some $z_{1} \in\left(0, z_{0}\right]$. Then there exists $T_{1}>0$ such that for all $R>0$ there exists a constant $C>0$ such that

$$
\left(G^{\prime}\right)^{-1}\left(\frac{R}{t}\right) \leq C z(t), \quad \forall t \geq T_{1},
$$

where $T_{1}$ is a positive constant.

Remark 2.8. The constant $C$ of the above Lemma depends explicitly on $\kappa, R$ (and in addition of $\mu$ if (2.27) holds). This dependence is given in the proof of Lemma 2.4 in [2].

Moreover, in the above Lemma, one may assume that $r_{0}=\infty$. In this case the interval $\left[0, r_{0}^{2}\right]$ becomes $[0, \infty)$ in the above Lemma.

Theorem 2.9. Assume the hypotheses of Theorem 2.3. Let $U_{0}=\left(\varphi^{0}, \psi^{0}, \varphi^{1}, \psi^{1}\right)$ be in $D\left(A_{i}\right)$. We denote by $U$ the solution of (2.1) and (2.6) subjected to (2.4) if $i=1$ and to (2.5) if $i=2$, and $E$ its energy. We assume in addition that either $\omega$ satisfies $(H S)$ or that $\lim _{t \longrightarrow \infty} E(t)=0$. We define $H$ and $\Lambda$ respectively by (2.11) and (2.13). We assume that

$$
(H 2)\left\{\begin{array}{l}
\exists r_{0}>0 \text { such that the function } H:\left[0, r_{0}^{2}\right] \mapsto \mathbb{R} \text { defined by }(2.11) \\
\quad \text { is strictly convex on }\left[0, r_{0}^{2}\right] \\
\text { and either } 0<\liminf _{x \rightarrow 0} \Lambda(x) \leq \lim \sup _{x \rightarrow 0} \Lambda(x)<1 \\
\text { or there exists } \mu>0 \text { such that } \\
0<\liminf _{x \rightarrow 0}\left(\frac{H(\mu x)}{\mu x} \int_{x}^{z_{1}} \frac{1}{H(y)} d y\right), \text { and } \lim \sup _{x \rightarrow 0} \Lambda(x)<1 \\
\text { for some } z_{1} \in\left(0, z_{0}\right] .
\end{array}\right.
$$


Then the energy $E$ satisfies the lower estimate

$$
\frac{1}{\gamma_{s}^{2} C_{\gamma_{s}}^{2}}\left(\left(H^{\prime}\right)^{-1}\left(\frac{1}{t-T_{0}}\right)\right)^{2} \leq E(t), \quad \forall t \geq T_{1}+T_{0},
$$

where $T_{0}$ is defined such as in Theorem 2.5 and $T_{1}$ such as in Lemma 2.7.

Proof. We use Lemma 2.7 as follows. We set $z_{0}=\gamma_{s} \sqrt{E\left(T_{0}\right)}$ and

$$
\kappa=\frac{c \alpha_{a}}{\rho_{2}}
$$

We denote by $z$ the solution of $(2.25)$ with $H$ replacing $G, \widehat{\Lambda}=\Lambda$. We set $\widehat{z}(t)=z\left(t-T_{0}\right)$ for $t \geq T_{0}$. Then we have

$$
\widehat{z}(t)=K_{s}^{-1}\left(\kappa\left(t-T_{0}\right)\right)=K_{s}^{-1}\left(\frac{c \alpha_{a}}{\rho_{2}}\left(t-T_{0}\right)\right), \quad \forall t \geq T_{0} .
$$

Thanks to $(H 2), \widetilde{H}$ is increasing on $\left[0, r_{0}^{2}\right]$, we can therefore apply Theorem 2.5. Hence (2.15) holds, so that we have

$$
\frac{1}{\gamma_{s}^{2}} \widehat{z}^{2}(t) \leq E(t), \quad \forall t \geq T_{0} .
$$

On the other hand applying Lemma 2.7 to $G=H$ for $R=1$, we deduce that there exists $C_{\gamma_{s}}>0$ depending on $\gamma_{s}$, and in addition of $\mu$ if (2.27) holds, such that

$$
\left(H^{\prime}\right)^{-1}\left(\frac{1}{t-T_{0}}\right) \leq C_{\gamma_{s}} \widehat{z}(t), \quad \forall t \geq T_{1}+T_{0} .
$$

Hence we have (2.29).

Theorem 2.10. Assume the hypotheses of Theorem 2.3. Let $U_{0}=\left(\varphi^{0}, \psi^{0}, \varphi^{1}, \psi^{1}\right)$ be in $D\left(A_{i}\right)$. We denote by $U$ the solution of (2.1) and (2.6) subjected to (2.4) if $i=1$ and to (2.5) if $i=2$, and $E$ its energy. We also assume that (H2) holds and that $\omega$ contains a neighbourhood of 0 and $L$. Then $E$ satisfies the lower estimate (2.29).

Proof. We use the stabilization results of Youssef [39] and Wehbe and Youssef [38] (see also the references therein). Thanks to these results, exponential stability holds in case of equal speeds of propagation $v_{1}$ and $v_{2}$, whereas polynomial stabilization holds for smoother initial data in case of different speeds of propagation. Hence in both cases, strong stabilization holds for initial data in the energy space, so that $E(t)$ converges to 0 as times goes to $\infty$. We can therefore apply Theorem 2.9 to conclude.

Remark 2.11. We conjecture that the above lower energy estimate holds true for arbitrary nonempty subset $\omega$ of $\Omega$.

The above result can also be deduced from [3] for globally distributed feedbacks, using the same argument as above. 
We now give examples of resulting lower energy estimates for examples of feedbacks. Many other examples can be given. We limit ourselves to these ones. One should note that the lower energy estimates given below, are not optimal in general.

Corollary 2.12. Assume the hypotheses of Theorem 2.9. Let $U_{0}=\left(\varphi^{0}, \psi^{0}, \varphi^{1}, \psi^{1}\right)$ be in $D\left(A_{i}\right)$. We denote by $U$ the solution of (2.1) subjected to (2.4) if $i=1$ and to (2.5) if $i=2$ and $E$ its energy. Then the following examples of lower energy estimates hold.

Example 1 (polynomial feedbacks)

let $g$ be given by $g(x)=x^{p}$ where $p>1$ on $\left(0, r_{0}\right]$.

Then we have the estimates

$$
c_{1} t^{-4 /(p-1)} \leq E(t)
$$

for an explicit positive constant $c_{1}$ which depends on $E(0)$ and $E_{1}(0)$ and for $t$ sufficiently large.

Example 2 (exponential feedbacks)

let $g$ be given by $g(x)=e^{-\frac{1}{x^{2}}}$ on $\left(0, r_{0}\right]$.

Then we have the estimates

$$
c_{1}(\ln (t))^{-2} \leq E(t)
$$

for an explicit positive constant $c_{1}$ which depends on $E(0)$ and $E_{1}(0)$ and for $t$ sufficiently large.

Example 3 (polynomial-logarithmic feedbacks)

let $g$ be given by $g(x)=x^{p}\left(\ln \left(\frac{1}{x}\right)\right)^{q}$ where $p>1$ and $q>1$ on $\left(0, r_{0}\right]$.

Then we have the estimates

$$
c_{1} t^{-4 /(p-1)}(\ln (t))^{-4 q /(p-1)} \leq E(t)
$$

for an explicit positive constant $c_{1}$ which depends on $E(0)$ and $E_{1}(0)$ and for $t$ sufficiently large.

Example 4 (between polynomial and exponential feedbacks) let $g$ be given by $g(x)=e^{-\left(\ln \left(\frac{1}{x}\right)\right)^{p}}$ where $1<p<2$ on $\left(0, r_{0}\right]$.

$$
c_{1} e^{-4(\ln (t))^{1 / p}} \leq E(t)
$$

for an explicit positive constant $c_{1}$ which depends on $E(0)$ and $E_{1}(0)$ and for $t$ sufficiently large.

\section{Strong lower energy estimates for Petrowsky equation in dimension two and three}

Let $\Omega$ be a bounded open subset of $\mathbb{R}^{N}$ with a boundary of class $\mathcal{C}^{4}$ denoted by $\Gamma$, where $N=2$ or $N=3$. We assume that $\omega$ is an open subset of $\Omega$ of 
positive measure. We consider the following Petrowsky equation with nonlinear damping:

$$
\begin{cases}\partial_{t t} u+\Delta^{2} u+a(.) g\left(u_{t}\right)=0 & \text { in } \quad(0, \infty) \times \Omega \\ u=0=\frac{\partial u}{\partial \nu} & \text { on } \Sigma=\Gamma \times \mathbb{R} \\ \left(u, \partial_{t} u\right)(0)=\left(u^{0}, u^{1}\right) & \text { on } \Omega,\end{cases}
$$

where $a \in L^{\infty}(\Omega)$ and $a \geq 0$ a.e. on $\Omega$ with $a>0$ on an open subset $\omega$ of $\Omega$ and where $g$ satisfies $(H 1)$ in all the sequel of this section.

The energy of a solution is defined by

$$
E(t)=\frac{1}{2}\left(\int_{\Omega}\left|u_{t}\right|^{2}+|\Delta u|^{2}\right) d x .
$$

We set $V=H_{0}^{2}(\Omega), H=L^{2}(\Omega)$ and $A u=\Delta^{2} u$ for $u \in D(A)$, where $D(A)$ is defined by $D(A)=H^{4}(\Omega) \cap H_{0}^{2}(\Omega)$.

Existence and regularity result hold in a classical way in $\mathcal{C}([0,+\infty) ; V) \times$ $\mathcal{C}^{1}([0,+\infty) ; H)$ (see e.g. [15] for the proof). Moreover if $\left(u^{0}, u^{1}\right) \in D(A) \times V$, the solution $u$ is in $L^{\infty}([0,+\infty) ; D(A)) \times W^{1, \infty}([0,+\infty) ; V) \times W^{2, \infty}([0,+\infty) ; H)$ and its energy satisfies the following dissipation relation:

$$
E^{\prime}(t)=-\int_{\Omega} a u_{t} g\left(u_{t}\right) d x \leq 0 .
$$

Moreover the energy of first order, defined by

$$
E_{1}(t)=\frac{1}{2}\left(\int_{\Omega}\left|u_{t t}\right|^{2}+\left|\Delta u_{t}\right|^{2}\right) d x,
$$

is nonincreasing.

We consider as for the Timoshenko system, a unique continuation result for the conservative system corresponding to (3.34), that is for

$$
\begin{cases}\partial_{t t} u+\Delta^{2} u=0 & \text { in } \quad(0, \infty) \times \Omega \\ u=0=\frac{\partial u}{\partial \nu} & \text { on } \quad \Sigma=\Gamma \times \mathbb{R},\end{cases}
$$

We assume now that $\omega$ is such that the following unique continuation result holds for weak solutions of (3.37)

$$
(H S P)\left\{u \text { is a solution of (3.37) and } u_{t} \equiv 0 \text { on } \omega \Longrightarrow u \equiv 0\right.
$$

Proceeding as in Dafermos [11] (see also the proof of Theorem 2.4), we prove

Theorem 3.1. Assume that $g$ satisfies $(H 1)$ and that $\omega$ satisfies $(H S P)$. Then for all $U_{0}=\left(u^{0}, u^{1}\right)$ in $V \times H$, the solution of (3.34) is such that its energy $E$ defined by (3.35) satisfies

$$
\lim _{t \longrightarrow \infty} E(t)=0
$$

We can establish a lower bound of the energy using comparison principles expressed through the energy of the solutions as in Sect. 2. 
Let $r$ be any real in $\left(N, q_{N}\right)$, where $q_{N}=\infty$ if $N=2$, whereas $q_{N}=6$ if $N=3$. We set

$$
\theta_{r}=\frac{1}{1+2 / N-2 / r}
$$

Then we recall the following Gagliardo-Nirenberg inequality

$$
\|v\|_{L^{\infty}(\Omega)} \leq C_{r}\|v\|_{L^{2}(\Omega)}^{1-\theta_{r}}\|v\|_{W^{1, r}(\Omega)}^{\theta_{r}} \quad \forall v \in W^{1, r}(\Omega)
$$

Theorem 3.2. Assume the hypotheses of Theorem 3.1. Let $U_{0}=\left(u^{0}, u^{1}\right)$ be in $\left(H^{4}(\Omega) \cap H_{0}^{2}(\Omega)\right) \times H_{0}^{2}(\Omega)$. We denote by $U$ the solution of $(3.34)$ and $E$ its energy. We assume in addition that either $\omega$ satisfies $(H S P)$ or that $\lim _{t \longrightarrow \infty} E(t)=0$. We define the functions $H$, and $\widetilde{H}$ respectively by $(2.11)$ and (2.12). We assume that $\widetilde{H}$ is nondecreasing on $\left[0, r_{0}^{2}\right]$ for $r_{0}>0$ sufficiently small. Let $r$ be any real in $\left(N, q_{N}\right)$ and $\theta_{r}$ be defined as in (3.39).

Then there exists $T_{0} \geq 0$ and $\gamma_{p}$, depending on $E_{1}(0)$ and $r$ such that defining $K_{p}$ by

$$
K_{p}(\tau)=\int_{\tau}^{\gamma_{p} E^{1-\theta_{r}}\left(T_{0}\right)} \frac{1}{H(y)} d y, \quad \tau \in\left(0, \gamma_{p} E^{1-\theta_{r}}\left(T_{0}\right)\right]
$$

E satisfies the lower estimate

$$
\left(\frac{1}{\gamma_{p}} K_{p}^{-1}\left(2 c \alpha_{a}\left(1-\theta_{r}\right)\left(t-T_{0}\right)\right)\right)^{1 /\left(1-\theta_{r}\right)} \leq E(t), \quad \forall t \geq T_{0},
$$

where $\alpha_{a}$ is defined in (2.23).

Moreover if $\lim _{\tau \rightarrow 0^{+}} K_{p}(\tau)=\infty$, then

$$
\lim _{t \rightarrow \infty} K_{p}^{-1}\left(c \alpha_{a}\left(1-\theta_{r}\right)\left(t-T_{0}\right)\right)=0
$$

so that the left hand side of (3.42) is converging to 0 as time goes to $\infty$.

Proof. Thanks to the smoothness of the solutions, the energy of first order of solutions of (3.34) is nonincreasing, so that

$$
E_{1}(t)=\frac{1}{2}\left(\int_{\Omega}\left|u_{t t}\right|^{2}+\left|\Delta u_{t}\right|^{2}\right) d x \leq E_{1}(0) \quad \forall t \geq 0 .
$$

This implies in particular, thanks to Sobolev embeddings that $u_{t} \in L^{\infty}([0, \infty)$; $W^{1, r}(\Omega)$ for any $r \in\left(N, q_{N}\right)$ and that $v=u_{t}$ satisfies (3.40), so that we have

$$
\left\|u_{t}(t)\right\|_{L^{\infty}(\Omega)}^{2} \leq \gamma_{p} E^{1-\theta_{r}}(t) \quad \forall t \geq 0,
$$

where $\gamma_{p}$ is a positive constant which depends on $r, N$ and $E_{1}(0)$.

If $E(t)$ converges to 0 when $t$ tends to $\infty$ or if (HSP) holds, then thanks to Theorem 3.1, there exists $T_{0} \geq 0$ such that

$$
E(t) \leq\left(\frac{r_{0}^{2}}{\gamma_{p}}\right)^{1 /\left(1-\theta_{r}\right)}, \quad \forall t \geq T_{0} .
$$

Thanks to $(3.36)$ and $(H 1)$, we have

$$
-E^{\prime}(t)=\int_{\Omega} a(x) u_{t} g\left(u_{t}\right) d x \leq c \alpha_{a} \int_{\Omega} u_{t}^{2} \widetilde{H}\left(u_{t}^{2}\right) d x \quad \forall t \geq T_{0},
$$


where $\alpha_{a}$ is defined by $(2.23)$. Thanks to our hypotheses, $\widetilde{H}$ is increasing on $\left[0, r_{0}^{2}\right]$, therefore we have

$$
\widetilde{H}\left(\left|u_{t}^{2}(t, .)\right|^{2}\right) \leq \widetilde{H}\left(\gamma_{p} E^{1-\theta_{r}}(t)\right) \quad \forall t \geq T_{0}, \quad x \in \Omega .
$$

Using this result in (3.46), we derive

$$
-E^{\prime}(t) \leq \frac{2 c \alpha_{a}}{\gamma_{p}} E^{\theta_{r}}(t) H\left(\gamma_{p} E^{1-\theta_{r}}(t)\right) .
$$

Thus we deduce that

$$
K_{p}\left(\gamma_{p} E^{1-\theta_{r}}(t)\right) \leq 2 c \alpha_{a}\left(1-\theta_{r}\right), \quad \forall t \geq T_{0} .
$$

Since $K_{p}$ is nonincreasing, we obtain (3.42).

As for Timoshenko beams, the above lower estimate can be characterized equivalently through pointwise time estimates, under some additional assumptions on $g$.

Theorem 3.3. Assume the hypotheses of Theorem 3.2. Let $U_{0}=\left(u^{0}, u^{1}\right)$ be in $\left(H^{4}(\Omega) \cap H_{0}^{2}(\Omega)\right) \times H_{0}^{2}(\Omega)$. We denote by $U$ the solution of (3.34) and $E$ its energy. We assume in addition that either $\omega$ satisfies (HSP) or that $\lim _{t \longrightarrow \infty} E(t)=0$. We define $H$ and $\Lambda$ respectively by (2.11) and (2.13). We assume that $(H 2)$ holds. Let $r$ be any real in $\left(N, q_{N}\right)$ and $\theta_{r}$ be defined as in (3.39). Then the energy E satisfies the lower estimate

$$
\left(\frac{1}{\gamma_{p} C_{\gamma_{p}}}\left(\left(H^{\prime}\right)^{-1}\left(\frac{1}{t-T_{0}}\right)\right)^{1 /\left(1-\theta_{r}\right)} \leq E(t), \quad \forall t \geq T_{1}+T_{0} .\right.
$$

Proof. We use Lemma 2.7 as follows. We set $z_{0}=\gamma_{p} E^{1-\theta_{r}}\left(T_{0}\right)$ and

$$
\kappa=2 c \alpha_{a}\left(1-\theta_{r}\right) .
$$

We denote by $z$ the solution of $(2.25)$ with $H$ replacing $G, \widehat{\Lambda}=\Lambda$. We set $\widehat{z}(t)=z\left(t-T_{0}\right)$ for $t \geq T_{0}$. Then we have

$$
\widehat{z}(t)=K_{p}^{-1}\left(\kappa\left(t-T_{0}\right)\right)=K_{s}^{-1}\left(2 c \alpha_{a}\left(1-\theta_{r}\right)\left(t-T_{0}\right)\right), \quad \forall t \geq T_{0} .
$$

Thus, thanks to (3.42), we have

$$
\left(\frac{1}{\gamma_{p}} \widehat{z}(t)\right)^{1 /\left(1-\theta_{r}\right)} \leq E(t), \quad \forall t \geq T_{0} .
$$

On the other hand applying Lemma 2.7 to $G=H$ for $R=1$, we deduce that there exists $C_{\gamma_{p}}>0$ depending on $\gamma_{p}$, and in addition of $\mu$ if (2.27) holds, such that

$$
\left(H^{\prime}\right)^{-1}\left(\frac{1}{t-T_{0}}\right) \leq C_{\gamma_{p}} \widehat{z}(t), \quad \forall t \geq T_{1}+T_{0} .
$$

Hence we have (3.47). 
We now assume the following geometric hypotheses on $\Omega$ and $\omega$ as in [26] (see also $[28,4]$ ) for use of the piecewise multiplier method:

$(H G)\left\{\begin{array}{l}\exists \varepsilon>0, \text { domains } \Omega_{j} \subset \Omega \text { with Lipschitz boundary } \Gamma_{j} \text { for } 1 \leq j \leq J \text { and points } x_{j} \text { in } \mathbb{R}^{N} \\ \text { such that } \Omega_{i} \cap \Omega_{j}=\emptyset \text { if } i \neq j, \\ \Omega \cap \mathcal{N}_{\varepsilon}\left[\cup_{j} \gamma_{j}\left(x_{j}\right) \cup\left(\Omega \backslash \cup_{j} \Omega_{j}\right)\right] \subset \omega,\end{array}\right.$ where $\gamma_{j}\left(x_{j}\right)=\left\{x \in \Gamma_{j},\left(x-x_{j}\right) \cdot \nu_{j}(x)>0\right\}$, and where the notation $\mathcal{N}_{\varepsilon}(O)$ stands for an $\varepsilon$ neighbourhood of a given set $O$ in $\mathbb{R}^{N}$.

Remark 3.4. These assumptions are a generalization of Zuazua's assumptions [40] for which one has $J=1$ and $\Omega_{1}=\Omega$.

Corollary 3.5. Assume the hypotheses of Theorem 3.3 and that $\omega$ satisfies $(H G)$. Let $r$ be any real in $\left(N, q_{N}\right)$ and $\theta_{r}$ be defined as in (3.39). Then $E$ satisfies the lower estimate (3.47). In particular for $N=2$, we have for any $r \in(2, \infty)$

$$
\left(\frac{1}{\gamma_{p} C_{\gamma_{p}}}\left(\left(H^{\prime}\right)^{-1}\left(\frac{1}{t-T_{0}}\right)\right)\right)^{1 /\left(1-\theta_{r}\right)} \leq E(t), \quad \forall t \geq T_{1}+T_{0},
$$

where $\theta_{r}$ tends to $1 / 2$ as $r$ goes to $\infty$.

Proof. We prove in [4] and [2] (see Theorem 4.10) that thanks to $(H G), E$ satisfies the upper estimate

$$
E(t) \leq C(E(0))\left(H^{\prime}\right)^{-1}\left(\frac{D}{t}\right)
$$

for $t$ sufficiently large and where $D$ is a positive constant which does not depend on $E(0)$ whereas $C(E(0))$ is a positive constant which depends on $E(0)$. Hence, strong stabilization holds, so that we can apply Theorem 3.3.

Remark 3.6. The constant involved in the lower estimate stated in Corollary 3.5 when $N=2$, depends on $r$ and blows up as $r$ goes to $\infty$.

The above results extend to the case for which the boundary conditions are replaced by $u=0=\Delta u$ on the boundary.

Corollary 3.5 can probably be generalized to weaker geometric assumptions using unique continuation results as stated in $(H S P)$ for weaker geometric assumptions.

Corollary 2.12 is valid for the energy of solutions of (3.34). We do not reformulate these examples of lower energy estimates for various examples of feedbacks.

We now state our weak lower velocity estimates

Theorem 3.7. Assume the hypotheses of Theorem 3.3 and that $\omega$ satisfies $(H G)$. Let $r$ be any real in $\left(N, q_{N}\right)$ and $\theta_{r}$ be defined as in (3.39). Then, the velocity satisfies the following weak lower estimate

$$
\limsup _{t \rightarrow \infty}\left(\frac{1}{\left(H^{\prime}\right)^{-1}\left(\frac{D_{0}}{t}\right)}\left\|u_{t}(t)\right\|_{L^{\infty}(\Omega)}^{2}\right) \geq 1 .
$$

We need the following lemma and corollary that we proved in [1]. 
Lemma 3.8. (Lemma $7.2[1])$ We assume that $g$ satisfies $(H 1)$ and that $g(0)=$ $g^{\prime}(0)=0$. We define $H$ as in (2.11) and $\widetilde{H}$ as in (2.12). We also define the function $\Lambda$ as in (2.13). We assume that $H$ satisfies (H2). We set $\mu=1$ if the first alternative of (H2) holds, otherwise $\mu>0$ is the one involved in the second alternative of $(H 2)$. Assume that $E:[0, \infty) \mapsto(0, \infty)$ is in $W_{l o c}^{1,1}([0, \infty)$ and satisfies the two inequalities

$$
-E^{\prime}(t) \leq \beta_{a} \widetilde{H}\left(\left\|u_{t}\right\|_{L^{\infty}(\Omega)}^{2}\right) E(t), \quad \forall t \geq T_{-},
$$

where $\widetilde{H}$ is defined in (2.12), $\beta_{a}>0$ and $T_{-} \geq 0$ are given constants, and

$$
E(t) \leq \beta_{E(0)}\left(H^{\prime}\right)^{-1}\left(\frac{D}{t}\right), \quad \text { for } t \text { sufficiently large. }
$$

Define the solution $z$ of the ordinary differential equation

$$
z^{\prime}(t)+H(\mu z(t))=0, \quad z(0)=z_{0}>0,
$$

where $z_{0}$ is given. Then we have

$$
\limsup _{t \rightarrow \infty}\left(\frac{\widetilde{H}\left(\left\|u_{t}(t)\right\|_{L^{\infty}(\Omega)}^{2}\right)}{\widetilde{H}(\mu z(t))}\right) \geq \frac{\mu}{\beta_{a}}>0 .
$$

Corollary 3.9. (Corollary 7.3 [1]) Assume the hypotheses of Lemma 3.8. Then, there exists $D_{0}>0$ depending on $\mu$ and $\beta_{a}$ such that

$$
\limsup _{t \rightarrow \infty}\left(\frac{1}{\left(H^{\prime}\right)^{-1}\left(\frac{D_{0}}{t}\right)}\left\|u_{t}(t)\right\|_{L^{\infty}(\Omega)}^{2}\right) \geq 1 .
$$

We now give the proof of Theorem 3.7.

Proof. Thanks to (3.46), $E$ satisfies (3.50) with $\beta_{a}=c \alpha_{a}$ and $T_{-}=T_{0}$. Moreover thanks to $(H G)$ and the results in $[2,4], E$ satisfies (3.48). Thus, we can apply Lemma 3.8, so that (3.53) holds. Applying Corollary 3.9, we deduce that (3.54) holds.

\section{Lower estimates for strong solutions of one-dimensional wave equations}

We improve our lower energy estimates obtained in [1], giving a sharper lower estimate.

We consider the nonlinearly damped wave equation

$$
\begin{cases}u_{t t}(t, x)-u_{x x}(t, x)+a(x) g\left(u_{t}(t, x)\right)=0, & 0<t, x \in \Omega, \\ u(t, c)=u(t, d)=0, & \text { for } 0<t, \\ u(0, x)=u_{0}(x), u_{t}(0, x)=u_{1}(x), & x \in \Omega,\end{cases}
$$

where $\Omega=(c, d) \subset \mathbb{R}$, with $-\infty<c<d<\infty, a \in L^{\infty}(\Omega)$ and $a \geq 0$ a.e. on $\Omega$ with $a>0$ on an open subset $\omega$ of $\Omega$, in all the sequel. Required existence results, monotonicity properties and appropriate a priori estimates either in 
monotonic situations or through compactness methods are contained in the works by Lions-Strauss [25] and further on in Lions's book [24].

The energy of a solution is defined by

$$
E(t)=\frac{1}{2} \int_{\Omega}\left(u_{t}^{2}+u_{x}^{2}\right) d x
$$

It is well-known that (4.55) is well-posed for initial data in the energy space $\mathcal{H}=H_{0}^{1}(\Omega) \times L^{2}(\Omega)$, i.e. for all $\left(u_{0}, u_{1}\right) \in \mathcal{H}$, (4.55) has a unique solution $u$ in $\mathcal{C}\left(\mathbb{R}_{+} ; H_{0}^{1}(\Omega)\right) \cap \mathcal{C}^{1}\left(\mathbb{R}_{+} ; L^{2}(\Omega)\right)$.

Moreover if $\left(u_{0}, u_{1}\right)$ is in $\left(H_{0}^{1}(\Omega) \cap H^{2}(\Omega)\right) \times H_{0}^{1}(\Omega)$ then $u \in \mathcal{C}\left(\mathbb{R}_{+} ; H_{0}^{1}(\Omega)\right.$ $\left.\cap H^{2}(\Omega)\right) \cap \mathcal{C}^{1}\left(\mathbb{R}_{+} ; H_{0}^{1}(\Omega)\right) \cap \mathcal{C}^{1}\left(\mathbb{R}_{+} ; L^{2}(\Omega)\right)$. Also the energy of higher order

$$
E_{1}(t)=\frac{1}{2} \int_{\Omega}\left(u_{t t}^{2}+u_{x t}^{2}\right) d x
$$

is well-defined, and nonincreasing. In this latter case, the natural energy $E$ satisfies the dissipation relation

$$
-E^{\prime}(t)=\int_{\Omega} a(x) u_{t}(t, x) g\left(u_{t}(t, x)\right) d x \quad t \geq 0 .
$$

Theorem 4.1. Assume that $\left(u_{0}, u_{1}\right) \in\left(H_{0}^{1}(\Omega) \cap H^{2}(\Omega)\right) \times H_{0}^{1}(\Omega)$ and that $g$ satisfies $(H 1)$. We define two functions $H$, and $\widetilde{H}$ respectively by (2.11) and (2.12). We assume that $\widetilde{H}$ is nondecreasing on $\left[0, r_{0}^{2}\right]$ for $r_{0}>0$ sufficiently small. Then there exists $T_{0} \geq 0$, depending on $E_{1}(0)$ such that defining $K_{\text {in }}$ by

$$
K_{i n}(\tau)=\int_{\tau}^{\gamma_{i n} \sqrt{E\left(T_{0}\right)}} \frac{1}{H(y)} d y, \quad \tau \in\left(0, \gamma_{i n} \sqrt{E\left(T_{0}\right)}\right]
$$

E satisfies the lower estimate

$$
\left(\frac{1}{\gamma_{i n}} K_{i n}^{-1}\left(\alpha_{a}\left(t-T_{0}\right)\right)\right)^{2} \leq E(t), \quad \forall t \geq T_{0},
$$

where $\alpha_{a}$ is defined in (2.23) and $\gamma_{\text {in }}$ is defined later in (4.62).

Moreover if $\lim _{\tau \rightarrow 0^{+}} K_{\text {in }}(\tau)=\infty$, then

$$
\lim _{t \rightarrow \infty} K_{i n}^{-1}\left(\alpha_{a} \quad\left(t-T_{0}\right)\right)=0,
$$

so that the left hand side of (4.60) is converging to 0 as time goes to $\infty$.

Proof. We proceed as for Timoshenko beams. We have

$$
u_{t}^{2}(t, x) \leq \gamma_{\text {in }} \sqrt{E(t)} \quad \forall t \geq 0, \quad \forall x \in \Omega .
$$

where

$$
\gamma_{i n}=4 \sqrt{E_{1}(0)} .
$$

Thanks to the dissipation relation (4.58) and the definition of $\widetilde{H}$, we have

$$
-E^{\prime}(t)=\int_{\Omega} a(x) u_{t}^{2} \widetilde{H}\left(u_{t}^{2}(t, x)\right) d x .
$$


Moreover, using Dafermos' Proposition 2.3 in [11], saying that $\lim _{t \rightarrow \infty} E(t)=0$, we deduce that there exists $T_{0} \geq 0$ such that $(2.21)$ holds. Thanks to our hypotheses, $\widetilde{H}$ is increasing on $\left[0, r_{0}^{2}\right]$. We thus have

$$
\widetilde{H}\left(\left|u_{t}(t, .)\right|^{2}\right) \leq \widetilde{H}\left(\gamma_{i n} \sqrt{E(t)}\right), \quad t \geq T_{0}, \text { in } \Omega .
$$

Using this inequality in (4.63), we obtain

$$
-E^{\prime}(t) \leq \widetilde{H}\left(\gamma_{i n} \sqrt{E(t)}\right) \int_{\Omega} a u_{t}^{2} d x \leq \frac{2 \alpha_{a}}{\gamma_{i n}} \sqrt{E(t)} H\left(\gamma_{i n} \sqrt{E(t)}\right) \quad t \geq T_{0} .
$$

Therefore, we deduce that

$$
K_{i n}\left(\gamma_{i n} \sqrt{E(t)}\right) \leq \alpha_{a}\left(t-T_{0}\right), \quad \forall t \geq T_{0}
$$

Since $K_{i n}$ is nonincreasing, we deduce the desired estimate.

Theorem 4.2. Assume that $\left(u_{0}, u_{1}\right) \in\left(H_{0}^{1}(\Omega) \cap H^{2}(\Omega)\right) \times H_{0}^{1}(\Omega)$ and that $g$ satisfies $(H 1)$. We define $H$ and $\widetilde{H}$ respectively as in (2.11) and (2.12). We also define $H$ and $\Lambda$ respectively as in (2.11) and (2.13). We assume that (H2) holds. Then the energy satisfies the lower estimate

$$
\frac{1}{\gamma_{i n}^{2} C_{\gamma_{i n}}^{2}}\left(\left(H^{\prime}\right)^{-1}\left(\frac{1}{t-T_{0}}\right)\right)^{2} \leq E(t), \quad \forall t \geq T_{1}+T_{0},
$$

where $\gamma_{i n}$ is defined in (4.62), and depends on $E_{1}(0)$, and $C_{\gamma_{i n}}$ depends explicitly on $\gamma_{i n}$, and $E_{1}(0)$.

Proof. We proceed as for the Timoshenko beams and use Lemma 2.7 as follows. We set $z_{0}=\gamma_{i n} \sqrt{E\left(T_{0}\right)}$ and $\kappa=\alpha_{a}$. We denote by $z$ the solution of (2.25) with $H$ replacing $G, \widehat{\Lambda}=\Lambda$. We set $\widehat{z}(t)=z\left(t-T_{0}\right)$ for $t \geq T_{0}$. Then we have

$$
\widehat{z}(t)=K_{\text {in }}^{-1}\left(\kappa\left(t-T_{0}\right)\right)=K_{\text {in }}^{-1}\left(\alpha_{a}\left(t-T_{0}\right)\right), \quad \forall t \geq T_{0} .
$$

Thus, thanks to (4.60), we have

$$
\frac{1}{\gamma_{\text {in }}^{2}} \widehat{z}^{2}(t) \leq E(t), \quad \forall t \geq T_{0}
$$

On the other hand applying Lemma 2.7 to $G=H$ for $R=1$, we deduce that there exists $C_{\gamma_{i n}}>0$ depending on $\gamma_{i n}$, and in addition of $\mu$ if (2.27) holds, such that

$$
\left(H^{\prime}\right)^{-1}\left(\frac{1}{t-T_{0}}\right) \leq C_{\gamma_{i n}} \widehat{z}(t), \quad \forall t \geq T_{1}+T_{0} .
$$

Hence we have (4.65).

Remark 4.3. Corollary 2.12 applies for the above example so that the corresponding energies satisfy the corresponding examples of lower estimates for the four examples.

Note that the lower bound obtained in [1] was

$$
\frac{1}{\gamma_{i n}^{2} C_{\gamma_{i n}}^{2}}\left(\left(\widetilde{H}^{\prime}\right)^{-1}\left(\frac{1}{t-T_{0}}\right)\right)^{2} \leq E(t), \quad \forall t \geq T_{1}+T_{0},
$$


We can see that the new lower bound given in (4.65) is strictly sharper for examples 1 and 3. We can give several further examples for which this lower estimate is strictly sharper than the one above. However for too weak dissipation, that is when the feedback $g$ converges to 0 as an exponential speed or close to an exponential speed as in examples 2 and 4 for instance, then the two lower estimates are equivalent.

\section{Improved lower bounds for locally damped wave equations in radial domains}

Following [1], we derive lower energy estimates for wave equations in annulus type domains in dimensions 2 and 3. We just adapt the results. The proofs with the improved lower bounds can easily be derived using the method in [1]. We formulate them here for the sake of completeness. Similarly to the one dimensional case, Corollary 2.12 applies for wave equations in radial domains of annulus type, so that the corresponding energies satisfy the corresponding examples of lower estimates for the four examples.

Locally distributed damping for wave equation in radial domains in $\mathbb{R}^{2}$. For the sake of simplicity, we will just consider a globally distributed case. The next results are obviously true in case of a localized feedback with a feedback coefficient which is radial and satisfies the same hypotheses in $\left(R_{1}, R_{2}\right)$ than in the one-dimensional case.

We assume that the space dimension is $N=2$ and we consider $\Omega=$ $B\left(0, R_{2}\right) \backslash B\left(0, R_{1}\right)$ in $\mathbb{R}^{N}$. We consider the equation

$$
\begin{cases}u_{t t}-\Delta u+g\left(u_{t}\right)=0, & 0<t, x \in \Omega, \\ u(t, .)=0, \text { on } \Gamma=\partial \Omega, & 0<t \\ u(0, x)=u_{0}(x), u_{t}(0, x)=u_{1}(x), & x \in \Omega .\end{cases}
$$

Well-posedness of (5.66) in the energy space $H_{0}^{1}(\Omega) \times L^{2}(\Omega)$ holds.

We restrict our attention to initial data that depend only on the radial component, so that we can extend our previous results. To avoid to introduce too many notation, we use the same notation for the radial function as a function on $\Omega$, and as a function of the radial component only.

Let $u_{0}()=.u_{0}(r)$ and $u_{1}()=.u_{1}(r)$ in $\Omega$ where $\left(u_{0}, u_{1}\right) \in\left(H_{0}^{1}\left(R_{1}, R_{2}\right) \cap\right.$ $\left.H^{2}\left(R_{1}, R_{2}\right)\right) \times H_{0}^{1}\left(R_{1}, R_{2}\right)$. Then the solution $u$ of (5.66) depends only on $r$. As above mentioned, we still denote this radial solution by $u$.

Then, $u$ satisfies the equation

$$
\begin{cases}r u_{t t}-\left(r u_{r}\right)_{r}+r g\left(u_{t}\right)=0, & 0<t, r \in\left(R_{1}, R_{2}\right), \\ u\left(t, R_{1}\right)=u\left(t, R_{2}\right)=0, & \text { for } 0<t \\ u(0, r)=u_{0}(r), u_{t}(0, r)=u_{1}(r), & r \in\left(R_{1}, R_{2}\right)\end{cases}
$$


Its energy is given by

$$
\begin{aligned}
E_{u}(t) & =\frac{1}{2} \int_{R_{1}}^{R_{2}} \int_{0}^{2 \pi}\left(u_{t}^{2}+\left|\partial_{r}(u)\right|^{2}\right) r d r d \theta \\
& =2 \pi E(t)=: 2 \pi \int_{R_{1}}^{R_{2}} \frac{1}{2}\left(u_{t}^{2}+u_{r}^{2}\right) r d r .
\end{aligned}
$$

We also define the energy of higher order

$$
\begin{aligned}
E_{1 u}(t) & =\frac{1}{2} \int_{R_{1}}^{R_{2}} \int_{0}^{2 \pi}\left(u_{t t}^{2}+\left|\partial_{r}\left(u_{t}\right)\right|^{2}\right) r d r d \theta \\
& =2 \pi E_{1}(t)=: 2 \pi \int_{R_{1}}^{R_{2}} \frac{1}{2}\left(u_{t t}^{2}+u_{t r}^{2}\right) r d r
\end{aligned}
$$

Theorem 5.1. Let $u_{0}()=.u_{0}(r)$ and $u_{1}()=.u_{1}(r)$ in $\Omega$ where $\left(u_{0}, u_{1}\right) \in$ $\left(H_{0}^{1}\left(R_{1}, R_{2}\right) \cap H^{2}\left(R_{1}, R_{2}\right)\right) \times H_{0}^{1}\left(R_{1}, R_{2}\right)$. Assume also that $g$ satisfies $(H 1)$. We define $H$ as in (2.11). We assume that (H2) holds.

Then the energy satisfies the lower estimate

$$
\frac{4 \pi}{\gamma^{2} C_{\gamma}^{2}}\left(\left(H^{\prime}\right)^{-1}\left(\frac{1}{t}\right)\right)^{2} \leq E_{u}(t), \quad \forall t \geq T_{1},
$$

where $\gamma$ is defined given by

$$
\gamma=\frac{4 \sqrt{E_{1}(0)}}{R_{1}}
$$

and $C_{\gamma}$ depends explicitly on $\gamma$ and $E_{1}(0)$.

Locally distributed damping for wave equation in radial domains in $\mathbb{R}^{3}$. As above and for the sake of simplicity, we will just consider a globally distributed case. The next results are obviously true in case of a localized feedback with a feedback coefficient which is radial and satisfies the same hypotheses in $\left(R_{1}, R_{2}\right)$ than in the one-dimensional case.

We assume that the space dimension is $N=3$ and we consider $\Omega=$ $B\left(0, R_{2}\right) \backslash B\left(0, R_{1}\right)$ in $\mathbb{R}^{N}$. We consider the equation (5.66). Well-posedness in the energy space $H_{0}^{1}(\Omega) \times L^{2}(\Omega)$ holds. For initial data that depend only on the radial component, we can extend our previous results.

Let $u_{0}()=.u_{0}(r)$ and $u_{1}()=.u_{1}(r)$ in $\Omega$ where $\left(u_{0}, u_{1}\right) \in\left(H_{0}^{1}\left(R_{1}, R_{2}\right) \cap\right.$ $\left.H^{2}\left(R_{1}, R_{2}\right)\right) \times H_{0}^{1}\left(R_{1}, R_{2}\right)$. Then the solution $u$ of (5.66) depends only on $r$.

Its energy with respect to spherical coordinates is given by

$$
E_{u}(t)=\frac{1}{2}\left(\int_{R_{1}}^{R_{2}} \int_{0}^{2 \pi} \int_{0}^{\pi}\left(u_{t}^{2}+\left|\frac{1}{r} \partial_{r}(r u)\right|^{2}\right) r^{2} \sin (\theta) d r d \theta d \phi\right) .
$$

We make the well-known change of unknown

$$
v(t, r)=r u(t, r), \quad t>0, \quad r \in\left(R_{1}, R_{2}\right) .
$$


Then, $v$ satisfies the equation

$$
\begin{cases}v_{t t}-v_{r r}+r g\left(\frac{v_{t}}{r}\right)=0, & 0<t, r \in\left(R_{1}, R_{2}\right), \\ v\left(t, R_{1}\right)=v\left(t, R_{2}\right)=0, & \text { for } 0<t \\ v(0, r)=r u_{0}(r), v_{t}(0, r)=r u_{1}(r), & r \in\left(R_{1}, R_{2}\right) .\end{cases}
$$

Moreover we have

$$
E_{u}(t)=4 \pi E(t)=: 4 \pi \int_{R_{1}}^{R_{2}} \frac{1}{2}\left(v_{t}^{2}+v_{r}^{2}\right) d r .
$$

Theorem 5.2. Let $u_{0}()=.v_{0}(r)$ and $u_{1}()=.v_{1}(r)$ in $\Omega$ where $\left(v_{0}, v_{1}\right) \in$ $\left(H_{0}^{1}\left(R_{1}, R_{2}\right) \cap H^{2}\left(R_{1}, R_{2}\right)\right) \times H_{0}^{1}\left(R_{1}, R_{2}\right)$. Assume also that $g$ satisfies $(H 1)$. We define $H$ as in (2.11). We also assume that (H2) holds.

Then the energy satisfies the lower estimate

$$
\frac{4 \pi}{\gamma_{i n}^{2} C_{\gamma_{i n}}^{2}}\left(\left(H^{\prime}\right)^{-1}\left(\frac{1}{t}\right)\right)^{2} \leq E_{u}(t), \quad \forall t \geq T_{1},
$$

where $\gamma_{i n}$ is defined in (4.62), and depends on $E_{1}(0)$, and $C_{\gamma}$ depends explicitly on $\gamma_{\text {in }}$ and $E_{1}(0)$.

Remark 5.3. A comparison principle for the energy can be stated as in the previous sections, but we do not formulate it here, to make the paper easier to read.

The previous results obviously extend to a damping term of the form $a(|x|) g\left(u_{t}\right)$ in (5.66), where $|\cdot|$ stands for the euclidian norm in $\mathbb{R}^{N}$, and where $a \in L^{\infty}\left(R_{1}, R_{2}\right), a \geq 0$ on $\left(R_{1}, R_{2}\right)$ and $a$ is nonvanishing on a subset of $\left(R_{1}, R_{2}\right)$.

\section{Conclusion and open questions}

The lower estimates given in the previous sections are not of the same order of convergence to 0 as time goes to $\infty$ than the sharp upper estimates given in $[1,2]$. More precisely, we recall that under classical geometric hypotheses on $\omega$ and for dampings which are not close to linear behavior close to the origin (see [2]), the energy of solutions of the wave, Petrowsky equations in dimensions two and three, or Timoshenko beams in case of equal velocities $v_{1}$ and $v_{2}$, satisfies the upper estimate

$$
E(t) \leq C(E(0))\left(H^{\prime}\right)^{-1}\left(\frac{1}{t}\right)
$$

for $t$ sufficiently large, and where $C(E(0))$ is a constant which depends on $E(0)$. We recall the lower energy estimate derived in Sects. 2, 4, 5 and a weaker estimate in Sect. 3 are

$$
C_{1}\left(E_{1}(0)\right)\left(\left(H^{\prime}\right)^{-1}\left(\frac{1}{t-T_{0}}\right)\right)^{2} \leq E(t),
$$

for $t$ sufficiently large, and where $C_{1}\left(E_{1}(0)\right)$ is a constant which depends on $E_{1}(0)$. Both the upper and lower bounds given above converges to 0 as times 
goes to $\infty$ but not at the same rate, so that these results are not sufficient to prove optimality.

Hence optimality is an open question. As said in the introduction, Vancostenoble [37], and Vancostenoble and Martinez [36] obtained first results in this direction for a one-dimensional boundary damped wave equation for initial data with vanishing velocity. We also give some optimality results in [5] applying their results. The question is open for general initial data, localized dampings, multidimensional domains. Similar questions hold for Petrowsky equation.

Another question which is of interest is the regularity of the solution of nonlinearly damped wave equations in case of arbitrary multidimensional domains. If solutions are such that $u_{t}$ is in $L^{\infty}\left([0, \infty) ; W^{1, p}(\Omega)\right)$ for a certain $2<p<\infty$ and where the a priori bound involves only the initial data, we proved in [2] a lower energy estimate for polynomial dampings. We proved in [1] that a stronger lower estimate holds if $p=\infty$, this for general dampings. This regularity holds in one-dimension as shown by Haraux [14], and in annulus domains in two and three dimensions. Is it possible to exhibit solutions which satisfy the prescribed regularity and a priori estimates for general multidimensional domains? We conjecture that such solutions may exist.

Also, for radial solutions in annulus domains, the lower estimate we derived does not depend on the dimension, whereas it depends on the dimension for general domains for solutions with $u_{t}$ in $L^{\infty}\left([0, \infty) ; W^{1, \infty}(\Omega)\right)$.

Furthermore, the answers on the above open questions may depend on characteristics of the initial data $[5,8]$. So it is important to keep trace on the dependence of the lower and upper estimates on the initial data. This question is also important in view of numerics and applications.

Another question of interest is the case of dissipations which are linear close to the origin and weak at infinity. These questions have been considered in $[18,23,30,32]$. In [30], the authors prove that if the globally distributed dissipation for a wave equation in two dimensional domains, is bounded or at most of polynomial growth at infinity, and linear close to the origin then the energy of strong solutions decays exponentially at infinity as $e^{-\omega t}$ where $\omega>0$ depends on a higher norm of the initial data. One can use the techniques of the present paper to give some lower bounds of exponential type in some situations. For instance, if $g(x)$ grows as $|x|^{q+1}$ for $|x| \geq 1$ and has a linear growth close to the origin, then one can easily show that in the case $q \in[0,1]$, there exists a positive constant $C$ independent of the initial data such that

$$
E(0) e^{-C t} \leq E(t) \quad \forall t \geq 0 .
$$

For this, one just needs to remark that thanks to the dissipation relation for strong solutions, one has

$$
-E^{\prime}(t)=\int_{\Omega} u^{\prime} g\left(u^{\prime}\right) \leq \frac{C}{2} \int_{\Omega}\left|u^{\prime}\right|^{2} \leq C E(t) \quad \forall t \geq 0
$$

where $C$ is such that

$$
0 \leq 2 x g(x) \leq C|x|^{2} \quad \forall x \in \mathbb{R} .
$$


A more involved result can also be obtained if $q>1$ for solutions with sufficiently smooth velocities, following our proofs in the present paper or in $[2,1]$. In this case, one also obtains a lower bound as above, but with a constant $C$ which depends on the energy of order 1 of the initial data. The general case, without such an a priori regularity result on the velocity is open. It would be also very interesting to derive lower energy estimates when the dissipation has a sublinear growth at the origin, and is superlinear at infinity.

Remark 6.1. The results given in this paper can easily be extended and adapted to dissipations of the form

$$
b(t) a(x) g\left(u_{t}\right)
$$

where $a$ is the damping coefficient for localization of the feedback and $b$ is a nonincreasing positive function on $[0, \infty)$.

\section{Acknowledgments}

We are grateful to the referees for their valuable comments and suggestions.

\section{References}

[1] Alabau-Boussouira, F.: New trends towards lower energy estimates and optimality for nonlinearly damped vibrating systems. J. Differ. Equ. 249, 1145-1178 (2010)

[2] Alabau-Boussouira, F.: A unified approach via convexity for optimal energy decay rates of finite and infinite dimensional vibrating damped systems with applications to semi-discretized vibrating damped systems. J. Differ. Equ. 248, 1473-1517 (2010)

[3] Alabau-Boussouira, F.: Asymptotic behavior for Timoshenko beams subject to a single nonlinear feedback control. Nonlinear Differ. Equ. Appl. 14(5-6), 643-669 (2007)

[4] Alabau-Boussouira, F.: Piecewise multiplier method and nonlinear integral inequalities for Petrowsky equations with nonlinear dissipation. J. Evol. Equ. 6(1), 95-112 (2006)

[5] Alabau-Boussouira, F.: Convexity and weighted integral inequalities for energy decay rates of nonlinear dissipative hyperbolic systems. Appl. Math. Optim. 51(1), 61-105 (2005)

[6] Alabau-Boussouira, F.: Une formule générale pour le taux de décroissance des systèmes dissipatifs non linéaires. C. R. Acad. Sci. Paris Sér I Math. 338, 35-40 (2004)

[7] Ammar-Khodja, F., Benabdallah, A., Munoz-Rivera, J.E., Racke, R.: Energy decay for Timoshenko systems of memory type. J. Differ. Equ. 194(1), 82-115 (2003) 
[8] Carpio, A.: Sharp estimates of the energy for the solutions of some dissipative second order evolution equations. Potential Anal. 1, 265-289 (1992)

[9] Conrad, F., Rao, B.: Decay of solutions of the wave equation in a star-shaped domain with nonlinear boundary feedback. Asymptot. Anal. 7, 159-177 (1993)

[10] Coron, J.-M.: Control and nonlinearity. In: Mathematical Surveys and Monographs, vol. 136. American Mathematical Society (2007)

[11] Dafermos, C.: Asymptotic behavior of solutions of evolution equations. In: Nonlinear Evolution Equations (Proc. Sympos., Univ. Wisconsin, Madison, Wis.). Publ. Math. Res. Center Univ. Wisconsin, vol. 40, pp. 103-123. Academic Press, New York (1978)

[12] Eller, M., Lagnese, J., Nicaise, S.: Decay rates for solutions of a Maxwell system with nonlinear boundary damping. Special issue in memory of Jacques-Louis Lions. Comput. Appl. Math. 21, 135-165 (2002)

[13] Fu, X., Yong, J., Zhang, X.: Exact controllability for multidimensional semilinear hyperbolic equations. SIAM J. Control Optim. 46, 1578-1614 (2007)

[14] Haraux, A.: $L^{p}$ estimates of solutions to some nonlinear wave equation in one space dimension. Publications du laboratoire d'analyse numérique, Université Paris VI, CNRS, Paris (1995). Published also under the same title in International Journal of Mathematical Modelling and Numerical Optimisation, vol. 1, 146-152. Inderscience Publishers (2009)

[15] Haraux, A.: Nonlinear evolution equations - global behavior of solutions. In: Lecture Notes in Mathematics, vol. 841. Springer-Verlag, Berlin (1981)

[16] Haraux, A.: Stabilization of trajectories for some weakly damped hyperbolic equations. J. Differ. Equ. 59, 145-154 (1985)

[17] Komornik, V.: Exact Controllability and Stabilization. The Multiplier Method. Collection RMA, vol. 36. Masson-John Wiley, Paris-Chicester (1994)

[18] Komornik, V.: Decay estimates for the wave equation with internal damping. In: Control and Estimation of Distributed Parameter Systems: Nonlinear Phenomena (Vorau, 1993), pp. 253-266. Internat. Ser. Numer. Math., vol. 118. Birkhäuser, Basel (1994)

[19] Lagnese, J.: Decay of solutions to wave equations in a bounded region. J. Differ. Equ. 50, 163-182 (1983)

[20] Lasalle, J.-P.: The extent of asymptotic stability. In: Proc. Symp. Appl. Math., vol. 13, pp. 299-307. Amunozsalva (1962)

[21] Lasalle, J.-P.: Asymptotic stability criteria. Proc. Natl. Acad. Sci. USA 46, 363$365(1960)$

[22] Lasiecka, I., Tataru, D.: Uniform boundary stabilization of semilinear wave equation with nonlinear boundary damping. Differ. Integr. Equ. 8, 507-533 (1993) 
[23] Lasiecka, I., Toundykov, D.: Stability of higher-level energy norms of strong solutions to a wave equation with localized nonlinear damping and a nonlinear source term. Control Cybernet. 36, 681-710 (2007)

[24] Lions, J.-L.: Quelques méthodes de résolution des problèmes aux limites non linéaires. Dunod, Gauthier-Villars, Paris (1969)

[25] Lions, J.-L., Strauss, W.: Some non linear evolution equations. Bull. Soc. Math. France 93, 43-96 (1965)

[26] Liu, K.: Locally distributed control and damping for the conservative systems. SIAM J. Control Optim. 35, 1574-1590 (1997)

[27] Liu, W.-J., Zuazua, E.: Decay rates for dissipative wave equations. Ricerche di Matematica 48, 61-75 (1999)

[28] Martinez, P.: A new method to obtain decay rate estimates for dissipative systems with localized damping. Rev. Mat. Complut. 12, 251-283 (1999)

[29] Martinez, P.: A new method to obtain decay rate estimates for dissipative systems. ESAIM Control Optim. Calc. Var. 4, 419-444 (1999)

[30] Martinez, P., Vancostenoble, J.: Exponential stability for the wave equation with weak nonmonotone damping. Portugal. Math. 57, 285-310 (2000)

[31] Munoz Rivera, J.E., Racke, R.: Mildly dissipative nonlinear Timoshenko systems-global existence and exponential stability. J. Math. Anal. Appl. 276(1), 248-278 (2002)

[32] Nakao, M.: Energy decay for the wave equation with a nonlinear weak dissipation. Differ. Integr. Equ. 8, 681-688 (1995)

[33] Nakao, M.: Decay of solutions of the wave equation with a local nonlinear dissipation. Math. Ann. 305, 403-417 (1996)

[34] Taylor, S.W.: Boundary control of the Timoshenko beam with variable physical characteristics. Research Report, Dept. Math. Univ. Auckland 356 (1998)

[35] Todorova, G., Yordanov, B.: The energy decay problem for wave equation with nonlinear dissipative terms in $\mathbb{R}^{n}$. Indiana Univ. Math. J. 56, 389-416 (2007)

[36] Vancostenoble, J., Martinez, P.: Optimality of energy estimates for the wave equation with nonlinear boundary velocity feedbacks. SIAM J. Control Optim. 39, 776-797 (2000)

[37] Vancostenoble, J.: Optimalité d'estimation d'énergie pour une équation des ondes amortie. C. R. Acad. Sci. Paris, 328, série I, 777-782 (1999)

[38] Wehbe, A., Youssef, W.: Stabilization of the uniform Timoshenko beam by one locally distributed feedback. Appl. Anal. 88, 1067-1078 (2009)

[39] Youssef, W.: Contrôle et stabilisation de systèmes élastiques couplés. Phd thesis, University Paul Verlaine-Metz (2009) 
[40] Zuazua, E.: Exponential decay for the semilinear wave equation with locally distributed damping. Commun. Partial Differ. Equ. 15, 205-235 (1990)

[41] Zuazua, E.: Uniform stabilization of the wave equation by nonlinear feedbacks. SIAM J. Control Optim. 28, 466-477 (1990)

[42] Zuazua, E.: Propagation, observation and control of wave approximation by finite difference methods. SIAM Rev. 47, 197-243 (2005)

F. Alabau-Boussouira

Present position Délégation

CNRS at MAPMO,

UMR 6628,

Orléans Cedex 2,

France

and

Université Paul Verlaine-Metz, LMAM UMR 7122,

Metz Cedex 1,

France

e-mail: alabau@univ-metz.fr

Received: 5 August 2010.

Accepted: 18 February 2011. 